\section{OPEN ACCESS}

Edited by:

Changiz Mohiyeddini,

Oakland University William Beaumont School of Medicine, United States

Reviewed by:

Livio Provenzi,

Neurological Institute Foundation Casimiro Mondino (IRCCS), Italy

Francesco Craig,

University of Calabria, Italy

*Correspondence:

Sara Calderoni

sara.calderoni@fsm.unipi.it

Specialty section:

This article was submitted to

Public Mental Health,

a section of the journal

Frontiers in Psychiatry

Received: 26 May 2020 Accepted: 26 October 2020 Published: 26 November 2020

Citation:

Conti E, Chericoni N, Costanzo V Lasala R, Mancini A, Prosperi M, Tancredi R, Muratori F, Calderoni S and Apicella F (2020) Moving Toward

Telehealth Surveillance Services for Toddlers at Risk for Autism During the

COVID-19 Pandemic.

Front. Psychiatry 11:565999. doi: 10.3389/fpsyt.2020.565999

\title{
Moving Toward Telehealth Surveillance Services for Toddlers at Risk for Autism During the COVID-19 Pandemic
}

\begin{abstract}
Eugenia Conti ${ }^{1}$, Natasha Chericoni ${ }^{1}$, Valeria Costanzo ${ }^{1}$, Roberta Lasala ${ }^{1}$, Alice Mancini ${ }^{1}$, Margherita Prosperi ${ }^{1,2}$, Raffaella Tancredi ${ }^{1}$, Filippo Muratori ${ }^{1,2}$, Sara Calderoni ${ }^{1,2 *}$ and Fabio Apicella ${ }^{1}$
\end{abstract}

${ }^{1}$ Department of Developmental Neuroscience - Istituto di Ricovero e Cura a Carattere Scientifico Fondazione Stella Maris, Pisa, Italy, ${ }^{2}$ Department of Clinical and Experimental Medicine, University of Pisa, Pisa, Italy

Since 2016, the project "Early Bird Diagnostic Protocol for Autism Spectrum Disorders (ASD)" funded by the Italian Ministry of Health has been operative at IRCCS Fondazione Stella Maris (FSM), Pisa (IT), with the main aim of developing early age-specific diagnostic protocols by longitudinally enrolling two different populations at risk for ASD: (i) toddlers with older siblings with ASD (FR) and (ii) toddlers referred by a child psychiatrist or pediatrician for suspected ASD (CR). On January 30, 2020, when the World Health Organization declared the outbreak of coronavirus disease 2019 (COVID-19), 136 patients (85 FR; 51 CR; 93 males; 43 females) had been enrolled in the project with 324 completed time points and 64 still missing. Considering both the huge psychological burden on families with toddlers at risk for ASD during the lockdown and the longitudinal studies reporting the positive "surveillance effect" in terms of a better outcome in at-risk toddlers, our priority has been to maintain regular contact and support to enrolled families. To do this, the research team, being authorized for smart-working research activities, has set up a detailed remote surveillance protocol (RSP). The RSP includes three online interviews and one online video registration of parent-child play. In the current community case study, the authors report the telehealth procedure and discuss possible future directions in developing remote assessment and new evaluation modalities for ecological parent-child play video recordings in at-risk populations. Hopefully, the surveillance protocol will further improve our ability to detect risk and activate early tailored intervention.

Keywords: autism spectrum disorders (ASD), coronavirus disease 2019 (COVID-19), public mental health, neurodevelopment, early identification, telehealth

\section{INTRODUCTION}

With an estimated prevalence of one in 87 children aged 7-9 years in Italy (1), autism spectrum disorders (ASD) are increasingly perceived as a public health priority, with a significant individual, familial, and societal burden, both emotional and economic. The phenotypic expression and the detrimental impact of ASD could be mitigated through early identification and intervention prior to the emergence of full-blown symptoms. Current literature, in fact, suggests that (i) it is possible 
to detect ASD starting from 14 months of age in at least a certain proportion of children (2); (ii) very young children benefit from early intervention (EI), especially when parents are actively involved in the rehabilitation process (3); and (iii) intervention should be initiated as soon as possible, when signs of ASD risk appear (4). Based on these three assumptions, since 2016 the project "Early Bird Diagnostic Protocol for Autism Spectrum Disorders" (EARLY BIRD; NET-2013-02355263-3) funded by the Italian Ministry of Health has been operative at IRCCS Fondazione Stella Maris (FSM), Pisa (IT), a tertiarycare University hospital that receives patients from all over Italy. This project aimed to develop, through longitudinal clinical monitoring and planned time points, age-specific diagnostic protocols able to detect (i) risk for ASD at 12 months of age; (ii) provisional diagnosis of ASD at 18 months of age; and (iii) stable diagnosis according to the diagnostic criteria of the DSM-5 (5) at 24 months of age. In addition, a clinical follow-up is scheduled at 30 months of age, to confirm the diagnosis or exclude it definitively. Children whose evaluations suggest a diagnosis of autism are referred to their local Child and Adolescent Mental Health Services (CAMHS) for early intervention.

A team of psychologists and child psychiatrists with many years of clinical and research experience in the ASD Unit of the FSM are in charge of the EARLY BIRD surveillance protocol of two different at-risk populations: (i) toddlers with older siblings with ASD (familial risk toddlers: FR), who are vulnerable to ASD and, more broadly, to psychiatric and neurodevelopmental disorders $(6,7)$ and (ii) toddlers clinically referred (CR) for suspected ASD by a child psychiatrist or pediatrician.

From September 2016 to January 2020, 136 children (85 FR; 51 TR; 93 males; 43 females) were enrolled in the study (see Table 1). A total of 106 subjects (FR: 58; CR: 48) have completed the diagnostic protocol, while 30 (FR: 27; CR: 3) are still being monitored. As part of the EARLY BIRD project, we completed 324 time points of the children enrolled, while 64 are still missing (5 at 12 months; 11 at 18 months; 18 at 24 months; 30 at 30 months).

In its original form, the EARLY BIRD surveillance protocol was conceived as a 2-day assessment of cognitive functioning, adaptive functioning, and social and communicative functioning, through standardized tests and interviews [Griffiths Mental Developmental Scales-ER (8), Vineland 2 (9), ADOS-2 (10), ADI-R (11)]. In addition, other information on each child's development was collected through parental questionnaires and clinical interviews (12-23) (see Table 2).

On January 30, 2020, the World Health Organization declared the outbreak of coronavirus disease 2019 (COVID-19) a public health emergency of international concern (24).

Emerging evidence reports the hard situation in which parents have been put during this unpredictable stressful situation, potentially impairing their ability to be supportive caregivers (25). Indeed, parents, especially those with young children, have to suddenly cope with closure of the kindergartens, worries over health and finances, the necessity for quick adaptation to "smartworking" at home, isolation, lack of support from grandparents, and bans on going to parks or other public spaces. Parents of toddlers at risk for ASD are even more exposed to stress, experimenting with concern that their child might have atypical development can impact family well-being and increase parents' risk of depression, ruminative thought, anxiety, or other types of psychological distress (26). In addition, stressful factors can interfere with early dyadic interaction between at-risk infants and their caregivers and, ultimately, play a detrimental role in children's longer-term social functioning and outcome (27). Some interesting suggestions about coping with young children with ASD in "stay at home period" are reported in Narzisi (28).

Coping difficulties may have even greater repercussions in families with infants who have an older sibling already diagnosed with ASD. Indeed, it is reported that caregivers of children with ASD have a higher risk of decreased family cohesion, depression, anxiety, somatic complaints, and burnout in comparison to caregivers of children with other developmental disabilities (29-32).

For all these reasons, our priority has been to maintain regular contact and support the families whose child was being followed in his developmental trajectory by the multidisciplinary team, in order to continue the active surveillance of toddlers at risk for ASD during the COVID-19 crisis. Indeed, a recent review of longitudinal studies on FR toddlers reported that the prospective follow-up strategy improves their developmental outcome, creating a sort of "surveillance effect" through which parents, who have the possibility to talk regularly about their child's development with clinical experts, can learn new strategies to interact with their high-risk infants, reducing, in turn, their symptom severity (33).

Despite technological tools and facilities (such as smartphones, tablets, PC, and wi-fi connections) spread and used among populations, there is little use for telehealthbased assessments of clinical conditions, including ASD, as recently systematically reviewed in Dahiya et al. (34). Obviously, the final goal consists of reaching the same percentage of success of the "in-person" assessment.

In a recent contribution, Juarez et al. (35) analyzed the accuracy of a remote diagnostic assessment for high-risk infants in the second-third year of life (the diagnosis was later confirmed with an in-person visit), using a remote video-analysis of a screening tool-the STAT (36-38). Despite that this diagnostic procedure succeeded in $62 \%$ of cases and the procedure was wellaccepted by parents, this was still dependent on the "physical" presence of a trained person who could administer the screening tool. On the contrary, Smith et al. (39) validated a novel remote procedure for ASD symptom assessment, where parents were instructed to elicit and record specific target behaviors in different scenarios, comparing it with an in-person assessment. Results indicated a high agreement $(88.2 \%)$ between the two modalities as well as high sensitivity (84.9\%) and high specificity (94.4\%). Following the same line, Sutantio et al. (40) found a good level of agreement ( $82 \%$ ) between parents' video recording, based on a given specific protocol, in three different home settings and a direct assessment conducted by an expert clinician.

In another study (41), Fusaro and colleagues interestingly examined the potential of applying the ADOS-G (42) protocol to unstructured homemade videos collected via the YouTube platform. They found that the ADOS-G-based video analysis 
TABLE 1 | Number of subjects at each time point (TP) are divided into completed (COM) and planned (PLA) protocol.

\begin{tabular}{|c|c|c|c|c|c|c|c|c|c|c|c|c|}
\hline & \multicolumn{2}{|c|}{ Subjects } & \multicolumn{2}{|c|}{ Total TP } & \multicolumn{2}{|c|}{$12 \mathrm{~m}$} & \multicolumn{2}{|c|}{$18 \mathrm{~m}$} & \multicolumn{2}{|c|}{$24 \mathrm{~m}$} & \multicolumn{2}{|c|}{$30 \mathrm{~m}$} \\
\hline & COM & PLA & COM & PLA & COM & PLA & COM & PLA & COM & PLA & COM & PLA \\
\hline CR & 48 & 3 & 106 & 3 & 4 & 0 & 29 & 0 & 41 & 0 & 32 & 3 \\
\hline $\mathrm{FR}$ & 58 & 27 & 218 & 61 & 57 & 5 & 61 & 11 & 55 & 18 & 45 & 27 \\
\hline TOT & 106 & 30 & 324 & 64 & 61 & 5 & 90 & 11 & 96 & 18 & 77 & 30 \\
\hline
\end{tabular}

CR, Clinical referral; FR, familial risk; TOT, Total.

achieved a classification accuracy of $96.8,94.1 \%$ of sensitivity, and $100 \%$ of specificity.

All these studies revealed that parents are capable of collecting appropriate examples of target behaviors of their children, especially when properly guided, and that these homebased procedures might improve the ecological validity of a diagnostic assessment (43). For this reason, we decided to adapt our original surveillance protocol to a remote procedure, aiming firstly to support families during the pandemic period and to provide, when necessary, feedbacks and advice on how to support child development or helping families to create a fast link to the local CAMHS.

Our efforts have been focused on instructing families on how to collect as much meaningful information as possible and to record an appropriate video recording of children's behavior. For this reason, differently from the previous studies on this topic, we have decided to be virtually present during the video recording, to promptly encourage parents to acquire appropriate examples of target behaviors.

This "perspective" paper describes how the protocol, already in use within the EARLY BIRD project, has been rethought and readjusted to be used remotely following the guidelines of health authorities, local governments and the World Health Organization in the COVID-19 era.

\section{Remote Surveillance Protocol}

The research team consists of three psychologists (V.C., F.A., and N.C.) and two child neurologists and psychiatrists (A.M. and R.T.). Due to the lockdown, all researchers have been authorized to continue activities in smart-working.

The team meets via video conference twice a week to discuss ongoing updates and issues, sharing the documents and materials for the remote surveillance protocol RSP within a shared folder on the cloud.

The RSP includes three online interviews and one online video registration of parent-child interaction in a playful setting, for a total of four online sessions. A preliminary telephone call is planned to inform families about the adapted telematic procedure and accept or decline participation. Afterward, caregivers who decide to participate in the remote evaluation fill in written informed consent procedures in compliance with institutional review board standards. Once informed consent has been acquired by the research team, parents are administered the following questionnaires by research assistants.

Detailed instructions on how to prepare the play area for the video recording are also provided. A minimum level of
TABLE 2 | The questionnaires administered to parents at each time point of the EARLY BIRD surveillance protocol.

\begin{tabular}{lcccc}
\hline & \multicolumn{3}{c}{ Time points (age of the child) } \\
\cline { 2 - 4 } & $\mathbf{1 2 \text { months }}$ & $\mathbf{1 8}$ months & $\mathbf{2 4}$ months & $\mathbf{3 0}$ months \\
\hline M-CHAT (12) & & $\checkmark$ & & \\
RBS-R (13, 14) & & $\checkmark$ & $\checkmark$ & $\checkmark$ \\
CBCL 1.5-5 (15) & & $\checkmark$ & $\checkmark$ & $\checkmark$ \\
ITSEA (16) & $\checkmark$ & $\checkmark$ & $\checkmark$ & $\checkmark$ \\
FYI (17) & $\checkmark$ & $\checkmark$ & $\checkmark$ \\
CDIS (18) & $\checkmark$ & $\checkmark$ & $\checkmark$ & $\checkmark$ \\
LUI (19) & & $\checkmark$ & $\checkmark$ & $\checkmark$ \\
EMQ (20) & $\checkmark$ & $\checkmark$ & $\checkmark$ & $\checkmark$ \\
PSI III (21) & $\checkmark$ & $\checkmark$ & $\checkmark$ & $\checkmark$ \\
QUIT (22) & $\checkmark$ & $\checkmark$ & $\checkmark$ \\
SP (23) & $\checkmark$ & & $\checkmark$ & $\checkmark$ \\
\hline
\end{tabular}

CBCL 1.5-5, Child Behavior Checklist; CDIs, MacArthur-Bates; Communicative Development Inventories; EMQ, Early Motor Questionnaire; FYI, First Year Inventory; ITSEA, Infant-Toddler Social and Emotional Assessment; LUI, Language Use Inventory; M-CHAT, Modified Checklist for Autism in Toddlers; PSI III, Parenting Stress Index, Third edition; QUIT, Italian Questionnaires of Temperament; RBS-R, Repetitive Behavior Scale-Revised; SP, Sensory Profile.

structure (e.g., in the selection of toys) is required in order to obtain informative video material, sufficiently comparable across children $(44,45)$.

\section{First Online Session}

The psychologist and the child psychiatrist meet the parents online to record $15 \mathrm{~min}$ of parent-child play. Parents are encouraged to resolve any doubts about the setup of the video registration before starting, taking advantage of the researchers' help. A picture of the setting is taken in order to facilitate the replicability of the video recording at future time points. Researchers follow the recording in "mute option," intervening only if necessary, to ensure the visibility of both parent and child.

In the days following the first session, the video is viewed and discussed by the researchers, focusing on those behaviors, included in the ADOS-2 Toddler Module (46) diagnostic algorithm, that does not need to be elicited by an adult: overall quality of the social overtures, eye contact, use of gestures to communicate, language level and prosody, play variety, joint attention behaviors, facial expressions, presence of unusual sensory interests, presence of repetitive movements and presence 
of restricted and repetitive patterns of behaviors and interests, and presence of other problematic behaviors. At the end of the discussion, a clinical hypothesis on the risk for ASD is made, as a starting point to be further examined during the following two sessions.

\section{Second Online Session}

An anamnestic interview or an anamnestic update is collected. Researchers also discuss with parents any concerns and information that emerged from questionnaires. Parents are asked to report recent auxological parameters (height, weight, and head circumference) as well as other general health status information. Vineland-II interview (9) with one parent is also carried out.

\section{Third Online Session}

The Socio-emotional Bayley III (47) interview is administered to parents along with a non-structured interview focused on age-appropriate behavioral risk signs for ASD or with the ADIR (48) (in the case of toddlers older than 24 months). Further information on the developmental level of the infant is collected through a non-structured interview based on the developmental milestones of play skills.

After the third session, the clinical-research team meets to discuss the information collected from the interviews and parental questionnaires, in order to decide if the child needs additional assessments to further investigate any causes for concern, which may have arisen. If not, the child will be evaluated again at his next time-point.

\section{Fourth Online Session}

The last session with parents includes feedback on the evaluations and discussion about developmental issues (if present). The appointment for the next time-point is then scheduled. Parents are also asked to compile a questionnaire to collect feedback on the remote procedure they have used including suggestions for improving the service.

If any kind of diagnosis or a consistent risk for ASD is hypothesized at one of the planned time points, the child's parents will be informed. At the same time, the local CAMHS will be contacted in order to start as soon as possible an early treatment tailored to specific strengths and challenges of the child.

A schematic representation of RSP is reported in Figure 1.

\section{CONSIDERATIONS}

Lockdown, which is often an unpleasant experience due to isolation, uncertainty about health and economical status with extensive effects on the general population (49), could have even worse consequences in those families with concerns about their child's development, thus forcing health workers to find out alternative remote strategies to keep in touch with them.

Preexisting evidence was already available about remote modality application in the ASD field either in assessment or in rehabilitation programs $(34,50)$, and during the COVID-19 emergency, the role of remote assistance in children with special needs has been even more stressed $(51,52)$.
The adaptation of the Autism Surveillance protocol reported here was systematized by the research team within the first few weeks of the Italian lockdown in order to do the following:

(1) maintain regular contact and support families involved in the longitudinal surveillance of their FR or CR toddler's development;

(2) evaluate the feasibility of an online surveillance protocol for toddlers at risk for autism and obtain evaluations at the time-points established by the project; and

(3) assess the level of compliance and satisfaction on the part of the families with regard to this new telematic protocol.

The current procedure emerged from multiple discussions among professionals with long experience in the field of early typical and atypical development. The main aim was to be able to perform an assessment, which was as structured and standardized as possible. One of the first challenges met was the impossibility to conduct a remote assessment of ASD symptoms and cognitive development using standardized tests such as the ADOS-Toddler and the Griffiths Mental Developmental Scales. To assess the child's spontaneous social behavior, a video depicting the child in interaction with the parent was collected. One of the most debated points regarding the procedure for the video registration was the level of structure. If, on the one hand, we wanted to collect clinically useful information, we did not want to stress parents by asking them to try to elicit particular behaviors (e.g., response to joint attention and imitation skills) as if they were clinicians. For this reason, we chose to ask parents to play with their child as they would naturally, but requested them to provide a specific selection of toys among those available in their house in order to elicit different levels of play and standardize the setting across different children. Despite these limitations, we are convinced that this could represent a way to experiment new procedures, potentially helpful in the future, to reach those patients who have difficulties getting to the hospital as has already been achieved in other countries (53).

Furthermore, the video recordings will also be helpful to assess parent-child interaction in their natural environment $(54,55)$. In order to obtain information on the children's development, we chose to investigate their level of play skills. For this purpose, we created a dedicated checklist that could be administered to parents during video-conference interviews.

Finally, we encouraged parents to comment on their experience of the telematic adaptation of the procedure and to share their level of satisfaction and perceived efficacy of the different web-platform appointments, highlighting any critical issues and providing suggestions where possible. It will provide researchers with precious feedback that will facilitate future improvements in remote procedures.

The preliminary feedback we have received since the start of the remote surveillance protocol suggests, in the majority of cases, a positive response to our proposal by the families, who have appreciated the possibility to keep in touch with the research team and to share any developmental concerns about their child. In addition, the telehealth procedure we have implemented allows families to avoid traveling to the FSM (they arrive from all over Italy), saving in this way time, money, time off from work, 


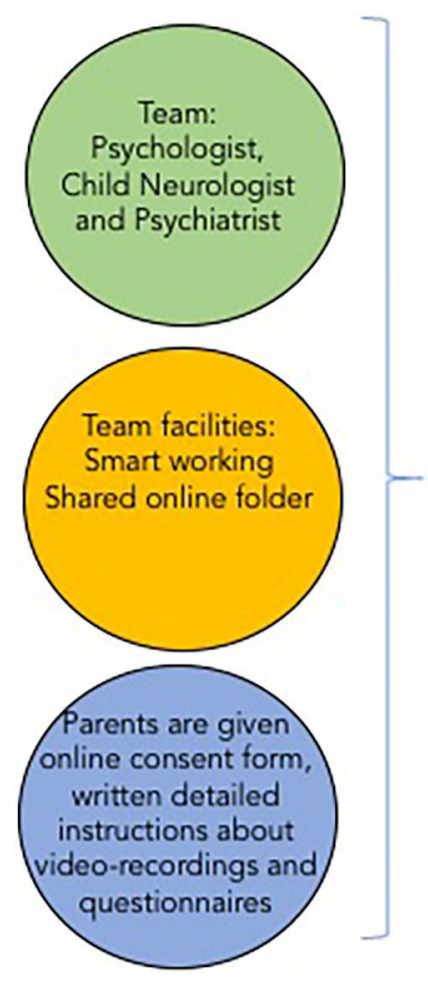

\section{Remote Surveillance Protocol (RSP)}

1st online session: 15 minutes online recording parent-child play<smiles>C1CCCCC1</smiles>

Team meeting: team discussion about ASD risk according to ADOS toddler<smiles>[13CH3]</smiles>

2nd online session: Hystory, clinical data and Vineland2 administration

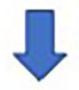

3rd online session: Socio-Emotional Bayley III Interview and ASD behavioural risk assessment

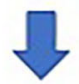

Team meeting: discussion about ASD risk on the data collection; decision point about necessity to switch to live visit according to risk rate

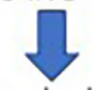

4th online session: parents feedback about online protocol and discussion about developmental concerns (if present).

FIGURE 1 | Schematic representation of the remote surveillance protocol (RSP).

and potentially stressful experience for both parents and child (e.g., due to travel meltdowns and sensory overload that could occur in the child).

\section{FUTURE DIRECTIONS}

Active surveillance of infants at risk for ASD should continue during COVID-19 social distancing restrictions in order to start an intensive intervention during early sensitive periods (56) for toddlers identified with atypical development. To this aim, we implemented the current protocol intended to provide telehealth assessment in at-risk populations.

We are fully aware that our telehealth assessment is not exhaustive and that the lack of remote standardized tests for ASD diagnosis could limit the ability to detect early risk signs of the disorder, especially with milder cases, leading us to be cautious in our diagnostic conclusions.

Unquestionable advantages of this procedure include (i) better access, especially for families with transportation and childcare challenges; (ii) the possibility of removing job-related absence as well as cost of the travel; and (iii) the opportunity to observe the child in their naturalistic environment and accordingly to provide more family-centered recommendations.

Conversely, extensive use of telehealth certainly needs the following: (i) appropriate knowledge about data security and data protection rules; (II) the use of user-friendly platforms that can be accessible by all caregivers regardless of their technological abilities or economic status; and (iii) the reduction of regulatory barriers that interfere with reimbursement for services provided via telehealth.

To overcome these difficulties, we used free platforms that are easily downloadable from any online store and do not require payment; we have also created a video tutorial to make downloads for parents. Through the online video registration, we have also avoided the telematic transmission of data by parents, reducing difficulties and risks deriving from this. Moreover, the informed consent contains a detailed section on data protection according to European regulation (GDPR 2016/679 (Prot. 4/2018 PO) and approved by our institutional Data Protection Officer.

In conclusion, in spite of negative health, social, and economic consequences, the current COVID-19 crisis could represent an opportunity to reorganize child mental health care by introducing innovative approaches through telehealth, thus paving the way for broader access and more efficient use of available public resources (57). Future investigations should accurately compare remote surveillance to face-toface evaluations (e.g., via randomized, controlled trials) in order to shed light on the efficacy, large-scale feasibility, and cost-effectiveness of online procedures, with the final aim of developing evidence-based guidelines for a virtuous coexistence of virtual and in-person assessment (58).

\section{DATA AVAILABILITY STATEMENT}

The raw data supporting the conclusions of this article will be made available by the authors, without undue reservation. 


\section{ETHICS STATEMENT}

The studies involving human participants were reviewed and approved by Pediatric Ethical Committee of Tuscany region at Meyer Children's Hospital. Written informed consent to participate in this study was provided by the participants' legal guardian/next of kin.

\section{AUTHOR CONTRIBUTIONS}

NC, VC, AM, RT, and FA conceived and created the protocol. FM supervised all the phases of the study. EC and SC wrote the paper. NC, VC, RL, AM, MP, FM, RT, and FA critically reviewed the manuscript. All authors contributed to the article and approved the submitted version.

\section{REFERENCES}

1. Narzisi A, Posada M, Barbieri F, Chericoni N, Ciuffolini D, Pinzino M, et al. Prevalence of autism spectrum disorder in a large italian catchment area: a school-based population study within the ASDEU project. Epidemiol Psychiatr Sci. (2018) 29:e5. doi: 10.1017/S2045796018000483

2. Landa RJ, Gross AL, Stuart EA, Faherty A. Developmental trajectories in children with and without autism spectrum disorders: the first 3 years. Child Dev. (2013) 84:429-42. doi: 10.1111/j.1467-8624.2012. 01870.x

3. Landa RJ. Efficacy of early interventions for infants and young children with, and at risk for, autism spectrum disorders. Int Rev Psychiatry. (2018) 30:25-39. doi: 10.1080/09540261.2018.1432574

4. Rogers SJ, Estes A, Lord C, Vismara L, Winter J, Fitzpatrick A, et al. Effects of a brief early start denver model (ESDM)-based parent intervention on toddlers at risk for autism spectrum disorders: a randomized controlled trial. J Am Acad Child Adolesc Psychiatry. (2012) 51:105265. doi: 10.1016/j.jaac.2012.08.003

5. American Psychiatric Association (2013). Diagnostic and Statistical Manual of Mental Disorders. 5th ed. Arlington, VA: American Psychiatric Association.

6. Charman T, Young GS, Brian J, Carter A, Carver LJ, Chawarska K, et al. NonASD outcomes at 36 months in siblings at familial risk for autism spectrum disorder (ASD): a baby siblings research consortium (BSRC) study. Autism Res. (2017) 10:169-78. doi: 10.1002/aur.1669

7. Jokiranta-Olkoniemi E, Cheslack-Postava K, Sucksdorff D, Suominen A, Gyllenberg D, Chudal R, et al. Risk of psychiatric and neurodevelopmental disorders among siblings of probands with autism spectrum disorders. JAMA Psychiatry. (2016) 73:622-9. doi: 10.1001/jamapsychiatry.2016.0495

8. Luiz BD, Knoesen DN. Administration Manual Griffiths Mental Developmental Scales-Extended Revised: Two to Eight Years. The Test Agency. Oxford: Hogrefe (2006).

9. Sparrow SS, Cicchetti DV, Balla DA. Vineland Adaptive Behavior Scales. 2nd ed. Circle Pines, MN: AGS Publishing (2005).

10. Lord C, Rutter M, DiLavore PC, Risi S, Gotham K, Bishop SL, et al. Autism Diagnostic Observation Schedule, Second Edition: ADOS-2. Torrance, CA: Western Psychological Services (2012).

11. Rutter M, Le Couteur A, Lord C. Autism Diagnostic Interview-Revised (ADIR) Manual. Los Angeles, CA: Western Psychological Services (2003).

12. Robins DL, Fein D, Barton ML, Green JA. The modified checklist for autism in toddlers: an initial study investigating the early detection of autism and pervasive developmental disorders. J Autism Dev Disord. (2001) 31:13144. doi: 10.1023/A:1010738829569

13. Bodfish JW, Symons FW, Lewis MH. The Repetitive Behavior Scale. Western Carolina Center Research Reports (1999).

14. Fulceri F, Narzisi A, Apicella F, Balboni G, Baldini S, Brocchini J, et al. Application of the repetitive behavior scale-revised-Italian version-in

\section{FUNDING}

This study was supported by the Italian Autism Spectrum Disorders Network: Filling the gaps in the National Health System care NET-2013-02355263. We are grateful to Università di Pisa for supporting MP with a research Grant (D.R. $354 \mathrm{n}$. 33134 29/05/2018). This work has been partially supported by grant from the IRCCS Fondazione Stella Maris (Ricerca Corrente, and the $5 \times 1000$ voluntary contributions, Italian Ministry of Health). EC and SC were partially funded by AIMS-2-Trials.

\section{ACKNOWLEDGMENTS}

The authors wish to thank all parents and children participating in this study.

preschoolers with autism spectrum disorder. Res Dev Disabil. (2016) 48:4352. doi: 10.1016/j.ridd.2015.10.015

15. Achenbach TM, Rescorla LA. Manual for the ASEBA Preschool forms and Profiles. Burlington, VT: University of Vermont; Research Center for Children, Youth, and Families (2000).

16. Briggs-Gowan MJ, Carter AS. Preliminary acceptability and psychometrics of the infant-toddler social and emotional assessment (ITSEA): a new adult-report questionnaire. Infant Ment Health J. (1998) 19:422-45. doi: 10.1002/(SICI)1097-0355(199824)19:4<422::AID-IMHJ5>3.0.CO;2-U

17. Baranek G, Watson LR, Crais E, Reznick S. First-Year Inventory (FYI) 2.0. Chapel Hill, NC: University of North Carolina (2003).

18. Fenson L, Marchman VA, Thal DJ, Dale PS, Reznick JS, Bates E. MacArthurBates Communicative Development Inventories: User's Guide and Technical Manual. 2nd ed. Baltimore, MD: Brookes (2007).

19. O'Neill DK. Language use inventory for young children: an assessment of pragmatic language development (Unpublished document). University of Waterloo, Waterloo, ON, Canada

20. Libertus K, Landa RJ. The early motor questionnaire (EMQ): a parental report measure of early motor development. Infant Behav Dev. (2013) 36:83342. doi: 10.1016/j.infbeh.2013.09.007

21. Abidin RR. Parenting Stress Index: Manual. 3rd ed. Odessa, FL: Psychological Assessment Resources (1995).

22. Axia G. QUIT. Questionari italiani del temperamento. Trento: Erickson Editore (2002).

23. Dunn W. Sensory Profile User's Manual. San Antonio, TX: Harcourt (1999).

24. World Health Organization. Statement on the Second Meeting of the International Health Regulations. Emergency Committee regarding the outbreak of novel coronavirus (2019-nCoV) (2020). Available online at: https://www.who.int/news/item/30-01-2020-statement-on-the-secondmeeting-of-the-international-health-regulations-(2005)-emergencycommittee-regarding-the- outbreak-of-novel-coronavirus-(2019-ncov)

25. Spinelli M, Lionetti F, Pastore M, Fasolo M. Parents' stress and children's psychological problems in families facing the COVID-19 outbreak in Italy. Front Psychol. (2020) 11:1713 doi: 10.3389/fpsyg.2020.01713

26. Haine-Schlagel R, Rieth S, Dickson KS, Brookman-Frazee L, Stahmer A. Adapting parent engagement strategies for an evidence-based parentmediated intervention for young children at risk for autism spectrum disorder. J Commun Psychol. (2020) 48:1215-37. doi: 10.1002/jcop.22347

27. Wan MW, Green J, Elsabbagh M, Johnson M, Charman T, Plummer F, et al. Quality of interaction between at-risk infants and caregiver at 12-15 months is associated with 3-year autism outcome. J Child Psychol Psychiatry. (2013) 54:763-71. doi: $10.1111 /$ jcpp. 12032

28. Narzisi A. Handle the autism spectrum condition during coronavirus (COVID-19) stay at home period: ten tips for helping parents and caregivers of young children. Brain Sci. (2020) 10:207. doi: 10.3390/brainsci100 40207 
29. Abbeduto L, Seltzer MM, Shattuck P, Krauss MW, Orsmond G, Murphy MM. Psychological well-being and coping in mothers of youths with autism, down syndrome, orfragile X syndrome. Am J Ment Retard. (2004) 109:237542. doi: 10.1352/0895-8017(2004)109<237:PWACIM>2.0.CO;2

30. Davis NO, Carter AS. Parenting stress in mothers and fathers of toddlers with autism spectrum disorders: associations with child characteristics. J Autism Dev Disord. (2008) 38:1278-91. doi: 10.1007/s10803-0070512-z

31. Ludlow A, Skelly C, Rohleder P. Challenges faced by parents of children diagnosed with autism spectrum disorder. J Health Psychol. (2012) 17:70211. doi: $10.1177 / 1359105311422955$

32. Weitlauf AS, Vehorn AC, Taylor JL, Warren ZE. Relationship satisfaction, parenting stress, and depression in mothers of children with autism. Autism. (2014) 18:194-8. doi: 10.1177/1362361312458039

33. Micheletti M, McCracken C, Constantino JN, Mandell D, Jones W, Klin A. Research review: outcomes of 24- to 36-month-old children with autism spectrum disorder vary by ascertainment strategy: a systematic review and meta-analysis. J Child Psychol Psychiatry. (2020) 61:4-17. doi: 10.1111/jcpp.13057

34. Dahiya AV, McDonnell C, DeLucia E, Scarpa A. A systematic review of remote telehealth assessments for early signs of autism spectrum disorder: video and mobile applications. Pract Innov. (2020) 5:150-64. doi: 10.1037/pri0000121

35. Juarez AP, Weitlauf AS, Nicholson A, Pasternak A, Broderick N, Hine J, et al. Early identification of ASD through telemedicine: potential value for underserved populations. J Autism Dev Disord. (2018) 48:260110. doi: 10.1007/s10803-018-3524-y

36. Stone WL, Coonrod EE, Ousley OY. Brief report: screening tool for autism in two-year-olds (STAT): development and preliminary data. J Autism Dev Disord. (2000) 30:607-12. doi: 10.1023/A:1005647629002

37. Stone WL, Coonrod EE, Turner LM, Pozdol SL. Psychometric properties of the STAT for early autism screening. J Autism Dev Disord. (2004) 34:691701. doi: 10.1007/s10803-004-5289-8

38. Stone WL, McMahon CR, Henderson LM. Use of the screening tool for autism in two-year-olds (STAT) for children under 24 months: an exploratory study. Autism. (2008) 12:557-73. doi: 10.1177/1362361308096403

39. Smith CJ, Rozga A, Matthews N, Oberleitner R, Nazneen N, Abowd G. Investigating the accuracy of a novel telehealth diagnostic approach for autism spectrum disorder. Psychol Assess. (2017) 29:245-52. doi: 10.1037/pas0000317

40. Sutantio JD, Pusponegoro HD, Sekartini R. Validity of telemedicine for diagnosing autism spectrum disorder: protocol-guided video recording evaluation. Telemed J E Health. (2020). doi: 10.1089/tmj.2020.0035. [Epub ahead of print].

41. Fusaro VA, Daniels J, Duda M, DeLuca TF, D'Angelo O, Tamburello $J$, et al. The potential of accelerating early detection of autism through content analysis of YouTube videos. PLoS ONE. (2014) 9:e93533. doi: 10.1371/journal.pone.0093533

42. Lord C, Rutter M, Goode S, Heemsbergen J, Jordan H, Mawhood L, et al. Autism diagnostic observation schedule: a standardized observation of communicative and social behavior. J Autism Dev Disord. (1989) 19:185212. doi: $10.1007 / \mathrm{BF} 02211841$

43. Nazneen N, Matthews N, Smith CJ, Rozga A, Abowd GD, Oberleitner R, et al. Use of a novel imaging technology for remote autism diagnosis: a reflection on experience of stakeholders. Proc Manufact. (2015) 3:293300. doi: 10.1016/j.promfg.2015.07.153

44. Grzadzinski R, Carr T, Colombi C, McGuire K, Dufek S, Pickles $\mathrm{A}$, et al. Measuring changes in social communication behaviors: preliminary development of the brief observation of social communication change (BOSCC). J Autism Dev Disord. (2016) 46:2464-79. doi: 10.1007/s10803-016-2782-9

45. Green J, Charman T, McConachie H, Aldred C, Slonims V, Howlin P, et al. Parent-mediated communication-focused treatment in children with autism (PACT): a randomised controlled trial. Lancet. (2010) 375:215260. doi: 10.1016/S0140-6736(10)60587-9

46. Luyster R, Gotham K, Guthrie W, Coffing M, Petrak R, Pierce K, et al. The autism diagnostic observation schedule-toddler module: a new module of a standardized diagnostic measure for autism spectrum disorders. J Autism Dev Disord. (2009) 39:1305-20. doi: 10.1007/s10803-009-0746-Z

47. Bayley N. Bayley Scales of Infant and Toddler Development. 3rd ed. San Antonio, TX: Harcourt Assessment (2006).

48. Lord C, Rutter M, Le Couteur A. Autism diagnostic interview-revised: a revised version of a diagnostic interview for caregivers of individuals with possible pervasive developmental disorders. J Autism Dev Disord. (1994) 24:659-85. doi: 10.1007/BF02172145

49. Brooks SK, Webster RK, Smith LE, Woodland L, Wessely S, Greenberg N, et al. The psychological impact of quarantine and how to reduce it: rapid review of the evidence. Lancet. (2020) 395:912-20. doi: 10.1016/S0140-6736(20)30460-8

50. Lindgren S, Wacker D, Suess A, Schieltz K, Pelzel K, Kopelman T, et al. Telehealth and autism: treating challenging behavior at lower cost. Pediatrics. (2016) 137(Suppl. 2):S167-75. doi: 10.1542/peds.2015-2851O

51. Provenzi L, Grumi S, Borgatti R. Alone with the kids: tele-medicine for children with special healthcare needs during COVID-19 emergency. Front Psychol. (2020) 11:2193. doi: 10.3389/fpsyg.2020.02193

52. Provenzi L, Borgatti R. Potentials of telerehabilitation for families of children with special health care needs during the coronavirus disease 2019 emergency. JAMA Pediatr. (2020). doi: 10.1001/jamapediatrics.2020.2351. [Epub ahead of print].

53. Johnsson G, Kerslake R, Crook S. Delivering allied health services to regional and remote participants on the autism spectrum via videoconferencing technology: lessons learned. Rural Remote Health. (2019) 19:5358. doi: 10.22605/RRH5358

54. Schreibman L, Dawson G, Stahmer AC, Landa R, Rogers SJ, McGee GG, et al. Naturalistic developmental behavioral interventions: empirically validated treatments for autism spectrum disorder. J Autism Dev Disord. (2015) 45:2411-28. doi: 10.1007/s10803-015-2407-8

55. Pickles A, Le Couteur A, Leadbitter K, Salomone E, Cole-Fletcher R, Tobin $\mathrm{H}$, et al. Parent-mediated social communication therapy for young children with autism (PACT): long-term follow-up of a randomised controlled trial. Lancet. (2016) 388:2501-9. doi: 10.1016/S0140-6736(16)31 229-6

56. Sullivan K, Stone WL, Dawson G. Potential neural mechanisms underlying the effectiveness of early intervention for children with autism spectrum disorder. Res Dev Disabil. (2014) 35:2921-32. doi: 10.1016/j.ridd.2014. 07.027

57. Figueroa CA, Aguilera A. The need for a mental health technology revolution in the COVID-19 pandemic. Front Psychiatry. (2020) 11:523. doi: 10.3389/fpsyt.2020.00523

58. Shore JH, Schneck CD, Mishkind MC. Telepsychiatry and the coronavirus disease 2019 pandemic-current and future outcomes of the rapid virtualization of psychiatric care. JAMA Psychiatry. (2020). doi: 10.1001/jamapsychiatry.2020.1643. [Epub ahead of print].

Conflict of Interest: The authors declare that the research was conducted in the absence of any commercial or financial relationships that could be construed as a potential conflict of interest.

Copyright (C) 2020 Conti, Chericoni, Costanzo, Lasala, Mancini, Prosperi, Tancredi, Muratori, Calderoni and Apicella. This is an open-access article distributed under the terms of the Creative Commons Attribution License (CC BY). The use, distribution or reproduction in other forums is permitted, provided the original author(s) and the copyright owner(s) are credited and that the original publication in this journal is cited, in accordance with accepted academic practice. No use, distribution or reproduction is permitted which does not comply with these terms. 